\title{
Phoenix rising from the ashes: A story of successful management of a severe paraquat poisoning.
}

\author{
${ }^{1}$ Thineskaran P, ${ }^{2}$ Selvaratnam $G,{ }^{2}$ Kumanan T, ${ }^{2}$ Suganthan $N$
}

${ }^{1}$ Teaching Hospital, Jaffna, ${ }^{2}$ Faculty of Medicine, Jaffna

\begin{abstract}
:
Self-poisoning with pesticides is a major public health problem in many developing countries, accounting for up to one-third of all suicides worldwide according to recent estimates and Sri Lanka is not an exception.
\end{abstract}

Here we reporting a case of paraquat poisoning in a 17-year-old-girl presented after self-ingestion. She developed oropharyngeal ulcers and clinical and laboratory evidence of multi organ damage. She was successfully treated with cyclophosphamide and steroid, initiated very early stage in addition to other supportive measurers. She made an uneventful recovery.

\section{Key words}

paraquat, superoxide, multi-organ dysfunction, cyclophosphamide

\section{Introduction}

Ingestion of paraquat is a common mode of deliberate self-harm.(1) Survival after acute paraquat poisoning is related to the ingested amount, the circumstances of poisoning, and the formulation ingested (2). A large amount of ingested paraquat ( $>40 \mathrm{ml} / \mathrm{kg}$ of $20 \%$ paraquat), vomiting after ingestion and age are considered to be important variables associated with high fatality rate in paraquat poisoning (2). Following ingestion of paraquat, it is concentrated inside many cells where it undergoes redox cycling, a process involving repetitive enzyme-mediated cycling between paraquat and paraquat radicals. A by-product of this process is a superoxide radical, a highly reactive oxygen species, which can cause direct cellular damage or react further to form other reactive oxygen species and nitrite radicals. Over a period of hours to days these processes would lead to multi-organ dysfunction or failure. The organs most affected are those with high blood flow, oxygen tension, and energy requirements, namely the lungs, heart, kidneys, and liver. (3)

\section{Case presentation}

A 17-year-old girl was transferred from a peripheral hospital following self-ingestion of paraquat taken 15 hours prior to admission. She had ingested around $10 \mathrm{ml}$ and got admitted to the peripheral hospital where the poison was identified by the attending medical officer as paraquat. She arrived to the local hospital within an hour. However, she was not treated with fuller's earth as it was not available. Subsequently she was transferred to the emergency treatment unit of our tertiary care centre. On arrival, she complained of severe burning pain of the throat and abdomen but denied difficulty in breathing. On admission her GCS was 15 and she was haemodynamically stable. She had several painful oral ulcers characterised by multiple coalescent ulcers with necrotic base involving oral mucosa and tongue (Figure 01). This led to swallowing difficulty since third day of ingestion. Complete blood count showed WBC-12.54 x 10 PLT- $388 \times 10^{3}$ and $\mathrm{Hb} 10.9 \mathrm{~g} / \mathrm{dL}$ with MCV 0 f $88 \mathrm{fL}$. Her initial renal profile was normal and the serum creatinine started to rise on day three and recorded a value of $311 \mathrm{microg} / \mathrm{dL}$ on the sixth day. Liver profile also started to deteriorate on day four and was totally deranged on the tenth day. The inflammatory markers were normal throughout her hospital stay. Adequate intravenous and gastrostomy fluid replacement was given throughout the hospital stay. Her vitals were monitored throughout. According to oro-maxillary and facial surgical team's opinion she was managed with a "cocktail mouthwash" with nystatin, dexamethasone, aspirin and tetracycline. Parenteral methylprednisolone was given on the outset for two days then changed to parenteral dexamethasone in a dosage of $20 \mathrm{mg}$ daily for three days and gradually tapered off with 
dexamethasone tablets. She received three courses of N-acetylcystine whenever she developed features of organ dysfunction. She received $1^{\text {st }}$ dose on day seven for alteration of renal function, on day ten for alteration of liver function and on day fourteen for the respiratory decompensation. In addition she was given parenteral cyclophosphamide $750 \mathrm{mg}$ after getting an informed written consent on day seven and eight when she developed acute kidney injury. She was also given parenteral antibiotics of cefuroxime and metronidazole to cover secondary infection following mucosal ulceration of gastroenteric system. Supportive therapy included an $\mathrm{H} 2$ receptor blocker, ranitidine and the anti-emetic ondansetron at appropriate dosage.

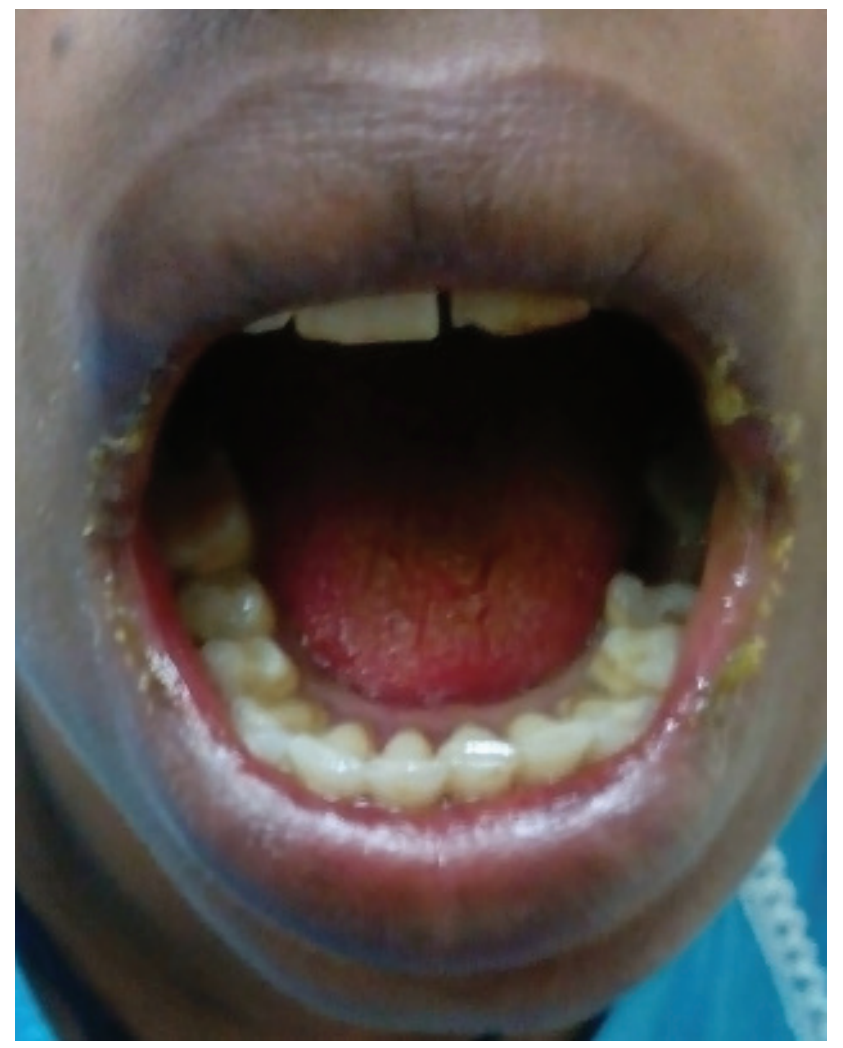

Figure 1: Characteristic 'paraquat tongue' with multiple coalescent ulcers with yellowish necrotic base.

\section{Discussion}

Paraquat is a rapidly-acting, nonselective herbicide that is relatively inexpensive. However, accidental or deliberate ingestion has an extremely high case-fatality rate. Largely for this reason, paraquat has been restricted in many parts of the world including Sri Lanka where it was completely banned in the year 2014. However few cases are encountered with paraquat poisoning which was thought to be purchased before it was banned. The brain is the organ that is uncommonly affected by paraquat as it does not readily cross the blood-brain barrier. Oral and gastrointestinal symptoms are common. Patients usually have a painful mouth and odynophagea. Nausea, vomiting, and abdominal pain occur in most patients. Respiratory complaints indicate systemic poisoning and are associated with fatal or near fatal outcomes. Measuring urine paraquat level may guide to assess severity of the exposure as well as outcome of poisoning. (5)

The management of paraquat poisoning is tailored to the victim depending upon the amount ingested and the time elapsed since the exposure. Overall, none of the current treatments has been proven to be effective for patients with severe poisoning and prognosis is uniformly poor in all centers throughout the globe, including those who were treated aggressively with multimodal and costly interventions and therapies. Gastrointestinal decontamination is recommended to limit systemic exposure. Hemoperfusion or hemodialysis followed by continuous hemodiafiltration or repeated hemoperfusion may be beneficial if commenced within four hours of poisoning. Many antidotes with logical mechanisms of action have been proposed, in particular anti-inflammatory and antioxidant therapies. However there are only limited animal and human data to support the efficacy of such treatments. Given the high mortality from acute paraquat poisoning, some centers administer all potentially beneficial treatment modalities to patients with acute paraquat poisoning in hope of obtaining some beneficial effect.

Resuscitation of the patient with acute paraquat poisoning follows standard guidelines except that oxygen therapy should not be administered unless there is confirmed hypoxia, as it may have an adverse impact on the oxygen-mediated cellular damage induced by redox cycling. Antiinflammatory and immunosuppressive therapy are the main stay of management, even though there are no proven large-scale trials. Parenteral dexamethasone and cyclophosphamide are commonly employed. Cases have been reported after subcutaneous injection and excision of the skin and subcutaneous tissue reported to save the life of a patient after paraquat ingestion (4). There are no evidence-based management strategies that exists to date for paraquat poisoning. Even though 
high dose immunosuppression is currently used for the management there are no convincing evidence to suggest that high dose immunosuppression improves survival in paraquat-poisoning (6). Antioxidants such as acetylcysteine and salicylate might be beneficial through free radical scavenging, anti-inflammatory and NF- $\kappa \mathrm{B}$ inhibitory actions. However, there are no published human trials to date. (7)

\section{References}

1. Bertolote JM, Fleischmann A, Eddleston M, Gunnell D (2006) Deaths from pesticide poisoning: a global response. Br J Psychiatry 189: 201-203

2. Fatality in paraquat poisoning: Sabzghabaee A M, Eizadi-Mood N, Montazeri K, Yaraghi A, Golabi M, Singapore Med J 2010; 51(6) : 496

3. Dinis-Oliveira RJ, Duarte JA, Sánchez-Navarro A, et al. Paraquat poisonings: mechanisms of lung toxicity, clinical features, and treatment. Crit Rev Toxicol 2008; 38:13-71.
4. Survival of a patient after self-injection of paraquat and surgical excision of the injection site R Fernando, Ceylon Medical Journal 2015; 60: 66

5. Paraquat Poisoning: Experience in Hospital Taiping (Year 2008 - October 2011) JenqTzong Tan, MRCP (UK), GR Letchuman Ramanathan, FRCP (London), Mun-Pung Choy, B Pharm, Leela Raman, MD, Boon-Kok Lim, MBBS

6. High-dose immunosuppression to prevent death after paraquat self-poisoning - a randomised controlled trial Indika Gawarammana, Nicholas A., Fahim Mohamed, Kamal Naser; , K. Jeganathan P. L. John A. Tomenson Martin F. Andrew H. Dawson

7. Medical management of paraquat ingestion, Indika B Gawarammana Nicholas A Buckley 